Conference Article

\title{
Tail Risk Assessment Using Support Vector Machine
}

\author{
O. Radović, J. Stanković and J. Stanković
}

University of Niš, Faculty of Economics, Serbia

\begin{abstract}
In this paper, authors apply Support Vector Regression (SVR) tool Oracle DM in forecasting volatility of Belex 15 index and estimation of Value-at-Risk (VaR). VaR is calculated using SVR model and compared to the results achieved implementing Markov Regime Switching model VaR and Feed Forward Neural Network VaR (ANN FFNN VaR). The results show that the SVR tools give better estimates of VaR comparing to other methods.
\end{abstract}

Keywords: Support Vector Regression, Value at Risk, Markov Regime Switching

\section{Introduction}

Artificial neural networks (ANN) models are widely used as computationally powerful tools for financial time series modeling. The advantage of their usage lies in their universal approximation capabilities any non-linear function arbitrarily well without a priori assumptions about the properties of the data (Hornik et al., 1989). Today, models based on support vector machine (SVM) algorithm are becoming increasingly popular. SVM is a special class of ANN algorithms developed by Vapnik (Vapnik, 1995, Vapnik et al., 1997). The advantage of SVM over traditional ANN is that SVM implements the structural risk minimization principle, while ANN minimizes the error on the in-sample data estimating. Therefore, SVM often give better results in predicting comparing to ANN (Tay and Lijuan, 2001; Sansom et al., 2003, Huang et al., 2004, Shin et al., 2005, Huang et al., 2005, Chen et al ., 2006). SVM is originally designed for classification problems; however, it is successfully used for regression problems. Gavrishchaka and Ganguli (2003) used SVM to predict volatility in foreign-exchange data, and show that the SVM gives better results than the GARCH model. Tests in computing Value at Risk in emerging financial markets showed that SVM outperforms traditional models and GARCH model (Stanković, Radović and Stanković, 2012).

\section{Research Data and Methodology}

In order to evaluate the performance of Support Vector Machines in tail risk assessment, we used stock index

\footnotetext{
*E-mail address: jelenas@eknfak.ni.ac.rs

ISSN: 1791-2377 (C) 20135Kavala Institute of Technology. All rights reserved.
}

BELEX15 as our reference portfolio. The financial time series of returns were obtained from the historical dataset provided by Belgrade Stock Exchange. The total number of dataset is 1125 observations divided in two sub-samples: insample of 750 observations and out-sample of 250 observations.

As it can be seen from the table 1, the summary statistics of the BELEX15 return series reveal significant kurtosis of the distribution and skewness, what indicates that distribution of this financial serial can not be considered normal. Results of the conducted tests indicate the positive serial correlation of returns and heteroscedasticity.

Table 1. Descriptive statistics of the Belex 15 index
\begin{tabular}{|l|l} 
& BELEX15 \\
& $(10.10 .2008-29.3 .2013)$ \\
Mean & $(1125)$ \\
Standard Deviation & -0.02023 \\
Min & 1.46956 \\
Max & -8.17977 \\
Skewness & 12.03364 \\
Kurtosis & 0.53224 \\
JB(5\%) & 11.65475 \\
& 3564.26995 \\
Q(12) & $(\mathrm{cv}=5.93678)$ \\
& $131.93383[0.46114]$ \\
Q ${ }^{2}(12)$ & $(\mathrm{cv}=21.02607)$ \\
& 343.93978 \\
ARCH(12) $\left.^{2}\right)$ & {$[0.000]$} \\
ARCH $^{2}(12)$ & $(\mathrm{cv}=21.02607)$ \\
& $249.28405[0.000]$ \\
& $(\mathrm{cv}=21.02607)$ \\
& $182.62408[0.000]$ \\
& $(\mathrm{cv}=21.02607)$
\end{tabular}

Note: JB is the Jarque-Bera normality test, $\mathrm{Q}(12)$ and $\mathrm{Q}^{2}(12)$ are 12-th orders Ljung-Box tests for serial correlation in the returns and squared returns. 
In estimation of daily returns volatility we have used Oracle Data Miner which includes a suite of SVM algorithms: SVM-classification (binary and multi-class), SVM-regression, and one-class SVM. Both Gaussian and Linear kernels are supported across all three classes of models.

We have used Gaussian kernel function on in-sample data, with $60 \%$ of data for building and $40 \%$ of data for testing model. Parameter values for SVR algorithm that provides best results for a given data set are presented in the table 3 .

Table 2. Variance forecast using Markov Switching Model

$\begin{array}{ll}\text { Switching Parameters } & \text { Switching } \\ \text { (Distribution Parameters) } & \text { Parameters } \\ & \text { (Regressors) }\end{array}$

$\begin{array}{lllll} & \text { State 1 } & \text { State 2 } & \text { State 1 } & \text { State 2 } \\ \text { Value } & 0.405442 & 3.248549 & - & 0.0816 \\ & & & 0.0198 & \\ \text { Standard } & 0.0392 & 0.4820 & 0.0313 & 0.1235 \\ \text { Error } & (0.00) & (0.00) & (0.53) & (0.51)\end{array}$

Transition Probabilities Matrix (std. error, p-value)

$\begin{array}{lll}\text { p1 } & 0.99 & 0.02 \\ & (0.20,0.00) & (0.20,0.91) \\ \text { p2 } & 0.01(0.00, & 0.98 \quad(0.00, \\ & 0.00) & 0.00)\end{array}$

Expected Duration of Regimes

Expected duration of Regime \#1: 89.26 time periods

Expected duration of Regime \#2: 42.82 time periods

Table 3. Parameter values for SVR algorithm

\begin{tabular}{ll}
\hline Parameter & Value \\
\hline Cutoff points & 5 Multiplies of sigma, \\
Missing values & $\begin{array}{l}\text { replace with edge values } \\
\text { Normalize }\end{array}$ \\
Split data & Z-score, min 0 , max 1 \\
& Build $60 \%$ Test $40 \%$, \\
Kernel function & Randomize before split \\
Tolerance value & Gaussian \\
Complexity factor & 0.001 \\
Active learning & No \\
Epsilon value & Yes \\
\hline
\end{tabular}

Using daily returns, we calculated VaR by historical simulation on out-sample data from 30 March, 2012 to 29 March, 2013 for a 1-day horizon, 99\%, 95\% and 90\% confidence level. In this analysis, we compare effectiveness of SVM to multilayer perception (MLP) neural network, as a universal function approximator, in assessing $\mathrm{VaR}$ of our reference portfolio. This type of architecture, MLP with back propagation (BP) learning, is the most frequently used to solve for curve adjustment or prediction; it is especially adequate for function adjustment and classification. (Aragones, Blanco and Garcia Estevez, 2005). In our study we use MLP neural network of the architecture 5-11-1 (5 input layer, 11 in hidden layer, and $1 \mathrm{i}$ output layer).

Following input variables have been selected for the network training:
- VaR (negative and positive), 100-day historical VaR (positive and negative) is the $95^{\text {th }}$ percentile of daily historical return distribution.

- Expected tail loss (ETL) and expected tail gain (ETG). Considering that each of the historical scenarios has equal probability of occurrence, the extreme tail values are calculated by taking a probability-weighted average of the losses (gains) beyond the VaR threshold.

- State 1 of Markov state switching model - state of low volatility,

- State 2 of Markov state switching model - state of high volatility,

- Conditional standard deviation calculated using Markov state switching model.

We computed the Markov state switching models (MS) with two states for which we saved the values of the probabilities for the series of the correlations to stay in one of the two states determined by the algorithm. The MS algorithm provided by Marcelo Perlin (2010) in Matlab was used in calculation. In order to investigate the regime switching change for both monetary and fiscal policies, we use Markov-switching regression method. According to Marcelo Perlin (2010), this method assumes that the transition of states is stochastic and not deterministic. This implies one is never sure whether there will be a switch of state or not. However, the dynamics behind the switching process is known and driven by a transition matrix. This matrix will control the probabilities of making a switch from one state to the other. The values of the probabilities for the series to stay in a certain state are recording the level of rigidity of the respective dynamics. The results of MS model are presented in Table 2 and Figure 1.
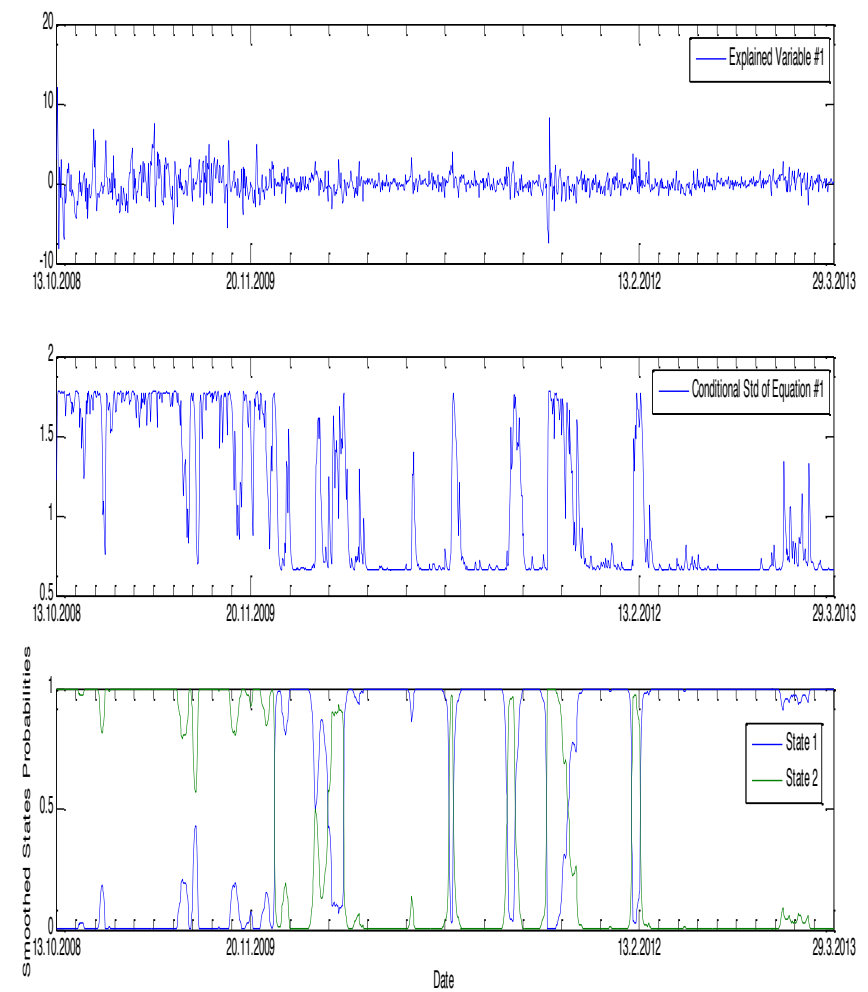

Fig. 1 Markov Regime Switching Model 
The output of our model based on SVR is 5-day observed $\mathrm{VaR}$ and the results are presented in the Table 4.

Table 4. Average VaR values at 90,95 and $99 \%$ confidence levels, for VaR models

\begin{tabular}{|c|c|c|c|}
\hline \multirow[t]{2}{*}{ var mode } & \multicolumn{3}{|c|}{ Average VaR } \\
\hline & $90 \%$ & $95 \%$ & $99 \%$ \\
\hline $\begin{array}{l}\text { Markov } \\
\text { Regime } \\
\text { Switching } \\
\text { VaR }\end{array}$ & $-0.86110 \%$ & $-1.11540 \%$ & $-1.59242 \%$ \\
\hline $\begin{array}{l}\text { Oracle } \\
\text { VaR }\end{array}$ & $-0.73374 \%$ & $-0.94175 \%$ & $-1.33193 \%$ \\
\hline $\begin{array}{ll}\text { ANN } & \text { FFNN } \\
\text { VaR } & \end{array}$ & $-0.77983 \%$ & $-1.00090 \%$ & $-1.41559 \%$ \\
\hline
\end{tabular}

\section{Results and Discussion}

The results on average VaR values at 90,95 and 99 percents confidence level are presented in Table 4. At all confidence level Oracle SVR VaR gives the lowest value of the average $\mathrm{VaR}$, likewise the lowest values for required capital at 90 and $95 \%$ confidence level, as it can be seen in Table 5 .

a)

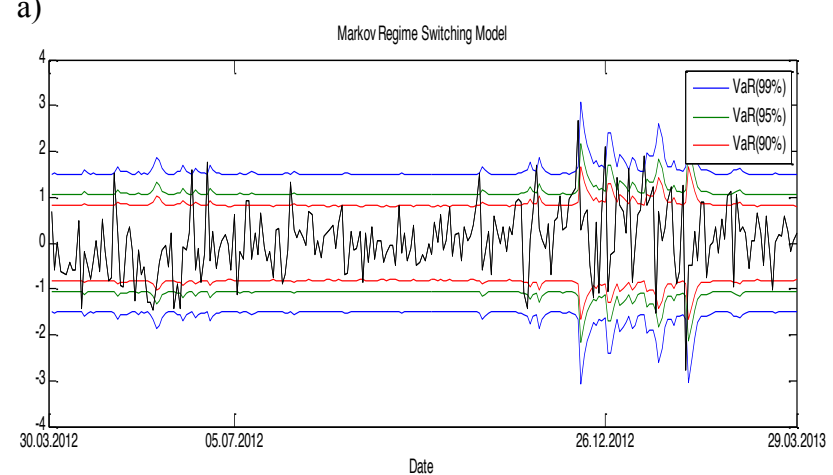

b)

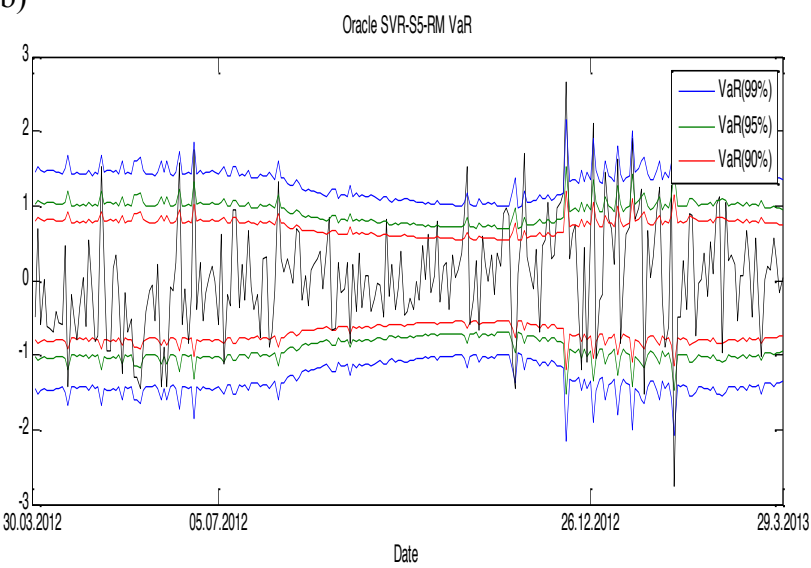

Fig. 2. The estimated value of the $\mathrm{VaR}$ for $90 \%, 95 \%$ and $99 \%$ confidence levels of the following models: a) Markov Regime Switching VaR, b) Oracle SVR VaR
Table 5. Capital requirements at 90,95 and $99 \%$ confidence levels, for VaR models

\begin{tabular}{|l|l|l|l|}
\cline { 2 - 4 } \multicolumn{1}{c|}{ Capital Requirements } \\
\cline { 2 - 4 } \multicolumn{1}{c|}{} & $90 \%$ & $95 \%$ & $99 \%$ \\
\hline $\begin{array}{l}\text { Markov Regime } \\
\text { Switching VaR }\end{array}$ & $3.44440 \%$ & $4.46160 \%$ & $4.77726 \%$ \\
\hline $\begin{array}{l}\text { Oracle SVR VaR } \\
\text { ONN FFNN VaR }\end{array}$ & $3.11931 \%$ & $4.00359 \%$ & $4.24676 \%$ \\
\hline ANN & & $3.76698 \%$ & $3.99578 \%$ \\
\hline & & & \\
\hline
\end{tabular}

There are a series of backtests based on the frequency of exceptions, such as the Kupiec (1995) or binomial test and the joint test suggested by Christoffersen (1998), which also takes into account the time independence of exceptions. However, those tests do not take into account the magnitude of those exceptions.

To identify what method of calculating $\mathrm{VaR}$ is acceptable to the regulators, we use Kupiec test at 5\% significance level for backtesting the achieved results. VaR values are estimated by setting a fixed window length and making a forecast one day in advance. Backtesting is implemented on the out-sample dataset by comparing achieved results with real data. The results of the overall acceptance, according to Kupiec test are presented in Table 6.

Table 6. Kupiec test backtesting results at 90,95 and $99 \%$ confidence levels

\begin{tabular}{|l|l|l|l|}
\cline { 2 - 4 } \multicolumn{1}{c|}{} & \multicolumn{2}{|l|}{ Kupiec test } \\
\cline { 2 - 4 } \multicolumn{1}{c|}{} & $90 \%$ & $95 \%$ & $99 \%$ \\
\hline $\begin{array}{l}\text { Markov Regime } \\
\text { Switching VaR }\end{array}$ & $\begin{array}{l}24 \\
(+)\end{array}$ & $\begin{array}{l}12 \\
(+)\end{array}$ & $\begin{array}{l}1 \\
(+)\end{array}$ \\
\hline Oracle SVR VaR & 31 & 15 & 2 \\
& $(-)$ & $(+)$ & $(+)$ \\
& & & \\
\hline ANN FFNN VaR & 28 & 15 & 4 \\
$(+)$ & $(+)$ & $(+)$ \\
& & & \\
\hline
\end{tabular}

The results of Kupiec test confirm acceptability of all tested methods for calculation VaR. The lowest value of average $\mathrm{VaR}$ and required capital is achieved by implementation of Oracle SVR VaR. However, according to the Kupiec test, Markov Regime Switching model VaR is acceptable at all level of confidence with the lowest number of exceptions. By training SVR model using different observed VaR (10-, 30-, 50-day) the level of average VaR is 
increasing, but the results of Kupiec test becomes similar to Markov Regime Switching model VaR.

\section{Conclusion}

The experimental results show that the SVM outperformed the Markov Regime Switching model and multilayer perception neural network. But day-by-day model updating provides more accurate forecasting. In this paper, we investigate settings of SVR parameters $(\mathrm{C}$, kernel), but we need to point out that forecasting performances of SVRs are sensitive to the value of the model parameters. This issue provides opportunities for significant improvement of the model. This study shows that SVR provides a promising alternative for forecasting financial time-series volatility.

Oracle's SVR implementation allows database users with relatively little knowledge on data mining tools with little effort in the use of tools to improve the SVR forecasting of financial time series and greatly improve the quality of the model based on these tools. However, the advance usage of implemented SVR tools is not an easy task and often depends on the user experience, which usually requires additional empirical research and the increased consumption of computing resources.

\section{References}

[1]. Aragonés, J. R., Blanco, C., \& Estévez, P. G. (2005). Improving expected tail loss estimates with neural networks. Intelligent Systems in Accounting, Finance and Management, 13(2), 81-94.

[2]. Chen WH, Jen-Ying S, Soushan WU (2006). Comparison of support vector machines and back propagation neural networks in forecasting the six major Asian stock markets. Int. J. Electron. Finan., 1: 49-67.

[3]. Gavrishchaka VV, Supriya BG (2003). Volatility forecasting from multiscale and high-dimensional market data. Neurocomputing, 55(1- 2): 285-305.

[4]. Hornik, K., Stinchcombe, M., \& White, H. (1989). Multilayer feedforward networks are universal approximators. Neural networks, 2(5), 359-366.

[5]. Huang W, Nakamori Y, Wang SY (2005). Forecasting stock market movement direction with support vector machine. Comput. Oper. Res., 32(10): 2513-2522.

[6]. Huang Z, Chen H, Hsu C-J, Chen W-H, Wu S ( 2004). Credit rating analysis with support vector machines and neural networks: a market comparative study. Decis. Support Syst., 37(4): 543-558.

[7]. Perlin, M. (2012). MS Regress-The MATLAB Package for Markov Regime Switching Models. Available at SSRN 1714016.
[8]. Sansom DC, Downs T, Saha TK ( 2003). Evaluation of support vector machine based forecasting tool in electricity price forecasting for Australian national electricity market participants. J. Electric Electron. Eng., 22(3): 227-234.

[9]. Stanković J, Stanković J, Radović O (2012). Using Oracle Data Miner for Financial Time Series Forecasting, Proceedings of the International Scientific Conference Serbia and European Union, Faculty of Economics, University of Nis, Serbia

[10]. Tay FEH, Lijuan C (2001). Application of support vector machines in financial time series forecasting. Omega, 29: 309317.

[11]. Vapnik V, Golowich S, Smola A (1997). Support vector method for function approximation, regression estimation, and signal processing, in: Mozer M, Jordan M, and Petsche T, (Eds.), Advances in Neural Information

[12]. Vapnik V. (1995). The nature of statistical learning theory, Springer-Verlag, New York.

[13]. Christoffersen PF. 1998. Evaluating interval forecasts. International Economic Revue 39: 841-862.

[14]. Kupiec P. 1995. Techniques for verifying the accuracy of risk management models. Journal of Derivatives 3:73-84. 